\title{
Review
}

Emmanuel J. Favaloro*, Leonardo Pasalic and Giuseppe Lippi

\section{Review and evolution of guidelines for diagnosis of COVID-19 vaccine induced thrombotic thrombocytopenia (VITT)}

https://doi.org/10.1515/cclm-2021-1039

Received September 24, 2021; accepted October 17, 2021;

published online November 1, 2021

\begin{abstract}
Coronavirus disease 2019 (COVID-19) is a lifethreatening infectious disease caused by Severe acute respiratory syndrome Coronavirus-2 (SARS-CoV-2). In response to the still ongoing pandemic outbreak, a number of COVID-19 vaccines have been quickly developed and deployed. Although minor adverse events, either local (e.g., soreness, itch, redness) or systematic (fever, malaise, headache, etc.), are not uncommon following any COVID-19 vaccination, one rare vaccine-associated event can cause fatal consequences due to development of antibodies against platelet factor 4 (PF4), which trigger platelet activation, aggregation, and possible resultant thrombosis, often at unusual vascular sites. Termed thrombosis with thrombocytopenia syndrome (TTS) by reporting government agencies, the term vaccine-induced (immune) thrombotic thrombocytopenia (VITT) is more widely adopted by workers in the field. In response to increasing reports of VITT, several expert groups have formulated guidelines for diagnosis and/or management of VITT. Herein, we review some key guidelines related to
\end{abstract}

*Corresponding author: Emmanuel J. Favaloro, PhD, FFSc (RCPA), Department of Haematology, Institute of Clinical Pathology and Medical Research (ICPMR), NSW Health Pathology, Westmead Hospital, Westmead, NSW 2145, Australia, Phone: +61 28890 6618, Fax: +61 296892331

E-mail: Emmanuel.Favaloro@health.nsw.gov.au. https://orcid.org/ 0000-0002-2103-1661

Leonardo Pasalic, Department of Haematology, Institute of Clinical Pathology and Medical Research (ICPMR), NSW Health Pathology, Westmead Hospital, Westmead, NSW, Australia; Sydney Centres for Thrombosis and Haemostasis, Westmead, NSW, Australia; and Sydney University, Westmead, NSW, Australia,

E-mail: Leonardo.Pasalic@health.nsw.gov.au. https://orcid.org/ 0000-0002-8237-6743

Giuseppe Lippi, Section of Clinical Biochemistry, University of Verona, Verona, Italy, E-mail: giuseppe.lippi@univr.it. https://orcid.org/ 0000-0001-9523-9054 diagnosis of VITT, and also provide some commentary on their development and evolution.

Keywords: diagnosis; laboratory testing; thrombosis with thrombocytopenia syndrome (TTS); guideline; vaccine induced thrombotic thrombocytopenia (VITT).

\section{Introduction}

Coronavirus disease 2019 (COVID-19) is a life-threatening infectious disease caused by Severe acute respiratory syndrome Coronavirus-2 (SARS-CoV-2), which has now become a pandemic. At time of writing, there have been over 240 million recorded infections worldwide, with nearly five million attributable deaths [1]. A large number of COVID-19 vaccines have been quickly developed as a response, with many also now deployed throughout the world to minimize the risk of severe COVID-19 and death, and also to prevent SARS-CoV-2 infection, including those caused by new "variants of concern". Of interest, a total of " 330 vaccine candidates are in various stages of development, with 102 in clinical research, including 30 in Phase I trials, 30 in Phase I-II trials, 25 in Phase III trials, and eight in Phase IV development" [2]. At time of writing, over 20 vaccines have been authorized by at least one national regulatory authority for public use: one DNA vaccine (ZyCoV-D), two RNA vaccines (Pfizer-BioNTech and Moderna), 10 conventional inactivated vaccines (BBIBP-CorV, Chinese Academy of Medical Sciences, CoronaVac, Covaxin, CoviVac, COVIran Barekat, FAKHRAVAC, MinhaiKangtai, QazVac, and WIBP-CorV), five viral vector vaccines (Sputnik Light, Sputnik V, Oxford-AstraZeneca, Convidecia, and Janssen), and five protein subunit vaccines (Abdala, EpiVacCorona, MVC-COV1901, Soberana 02, and ZF2001) [2, 3].

Minor adverse effects are an expected consequence of any vaccination program. For COVID-19 vaccines, these include soreness, redness, rash, and inflammation at the injection site. Other common side effects include fever, fatigue, headache, myalgia (muscle pain), and arthralgia 
(joint pain), which generally resolve within a few days [2, 3]. A less-frequent side effect (that generally occurs in less than one in 1,000 people) is hypersensitivity (allergy) to one or more vaccine ingredients, which in some rare cases may cause anaphylaxis. An ever-rarer side effect (that generally occurs in less than one in 50,000 people), now recognized to occur following immunization with adenovirus-based vaccines, has been termed thrombosis with thrombocytopenia syndrome (TTS), for TTS, by government reporting agencies, including the Centers for Disease Control (and Prevention) (CDC) in the US [4], the European Medicines Agency (EMA) [5], and the Therapeutic Goods Administration (TGA) in Australia [6].

However, the term vaccine-induced (immune) thrombotic thrombocytopenia (VITT) is more widely adopted by workers in the field [7], and will generally be used in this review, which is aimed to review the key guidelines related to diagnosis of this condition, and also provide some commentary on their development and evolution.

\section{A brief early history of VITT identification}

VITT was first reported in a non-reviewed preprint [8], by a German group of researchers led by Andreas Greinacher, and which was later used as the basis for a formal peer reviewed publication in the New England Journal of Medicine (NEJM) [9]. The German researchers originally called the syndrome VIPIT, for vaccine-induced prothrombotic immune thrombocytopenia [8], but later used the term VITT [9], as did two other European groups publishing also at that time in the NEJM, one from Norway [10], and the other from the United Kingdom [11]. All three publications reported on case series, respectively 11,5 , and 23 cases, and all associated to use of the adenovirus-based AstraZeneca (AZ) vaccine (alternatively called ChAdOx1 nCoV-19, AZD1222, Vaxzevria). Separate cases were later described in the USA, as associated to use of the adenovirus-based Janssen (Johnson \& Johnson) vaccine (JJ; alternatively known as Ad26.COV2.S or JNJ-78436735) [12, 13]. A subsequent review by one of us detailed some findings from the composite of approximately 100 cases of VITT published in the literature by the end of May, 2021 [7], with all cases associated with either the adenovirus-based $\mathrm{AZ}$ or JJ vaccines. Despite availability of other adenovirus-based vaccines $[2,3]$, the authors remain unaware of any substantiated reports of VITT/TTS by any other adenovirusbased COVID-19 vaccine, which may be due to lower worldwide usage of other formulations. Whether this actually reflects an absence of events or an absence of reporting is also unknown. We are aware, however, of a single case of potential VITT associated with use of the Moderna (mRNA-1273) vaccine [14], with this publication accompanied by an editorial [15]. Although this report fits a potential VITT/TTS case definition, and was positive for anti-PF4 antibodies, the test was performed after initiation of heparin treatment, so that such case might alternatively reflect a heparin-related anti-PF4 antibody, or even might be co-incidental and reflect a kind of "spontaneous HIT syndrome". In summation, only the use of AZ and JJ COVID-19 vaccines have consistently led to reports of vaccine associated VITT/TTS.

Common to patient presentation in all case series, was thromboses, usually at atypical sites, and for laboratory testing, positivity for the presence of platelet factor 4 (PF4) antibodies detected by enzyme linked immunosorbent assays (ELISA) [7]. Of interest, these assays are normally used to identify PF4/heparin $(\mathrm{PF} 4 / \mathrm{H})$ antibodies present in a potentially "related" condition called heparin induced thrombocytopenia (HIT) [16]. Of additional interest, other rapid assays normally used to identify $\mathrm{HIT} \mathrm{PF} 4 / \mathrm{H}$ antibodies [16-18] were usually negative in VITT [7]. Thus, although the VITT syndrome has been identified to be mechanistically similar to HIT, the antibodies did not appear to be the same as those seen in HIT. More specifically, VITT antibodies were found to bind some surface PF4 amino acids, specifically located within the heparin binding site, but differing from the protein moiety recognized by anti-PF4 antibodies which develop in HIT patients [19]. The consequent pathogenetic process of VITT is therefore partially different from HIT, in that immune complexes independent of heparin are generated in patients with VITT; these then promote cross-linking of FcyRIIa receptors on platelet surface, which is then followed by platelet activation and aggregation [19].

Differences have also been observed in functional assays looking for platelet activation and aggregation [7]. For example, inclusion of heparin in standard serotonin release assays (SRAs) is used to identify heparin-associated platelet activation and aggregation in HIT, but inclusion of heparin in SRA testing of VITT patients appeared to more often inhibit (rather than augment) platelet activation and aggregation $[7,9,20]$, according to the aforementioned differing molecular target(s). This therefore offered a potential for differentiation of HIT vs. VITT [7].

On the other hand, it is also important to recognize the potential for false negative functional assays in VITT/TTS investigations, should blood be collected after initiation of IVIG, this being a recognized and important early treatment in course of VITT/TTS, to both inhibit platelet 
activation and help return platelet counts to "normal" baseline values [21].

Irrespective of the above considerations, given the potentially serious consequences seen in a proportion of patients with VITT/TTS, several expert groups were quick to provide guidance on both the diagnosis and management of VITT; however, the current review details the key guidelines related to diagnosis of VITT.

\section{Guidelines from Europe}

Considering that VITT was first described in a German cases series, followed quickly by cases from Norway and the UK, it is fitting to begin with a review and comparison of diagnostic guidelines across Europe. One of the first "expert group" guidelines to appear was from the Society for Thrombosis and Haemostasis Research (GTH [Gesellschaft für Thrombose- und Hämostaseforschung]), a nonprofit, interdisciplinary German-Austrian-Swiss scientific society with the aim of promoting the scientific interests of blood coagulation research [22]. This publication included an algorithm that provided guidance for both identification and management of VITT, that appears to be partially modeled on that published by the German group in NEJM [8]. The portion related to the GTH diagnostic pathway is summarized in Figure 1, but their algorithm had the primary aim to provide appropriate management according to the diagnostic pathway findings. The key features are: exposure to the $\mathrm{AZ}$ vaccine (the only adenovirus-based vaccine available in the GTH countries) 4-16 days earlier, followed by evidence of thrombosis and thrombocytopenia; a "HIT screening test for PF4/H antibodies" would then be performed (with the following qualification: "Based on preliminary observations, the HYPHEN BioMed ZYMUTEST and the Immucor GTI Diagnostics enzyme immunoassays appear to have appropriate sensitivity for all pathophysiologically relevant antibodies"). If the test is negative, this essentially "excluded" VITT (or HIT), and thus treatment with heparin would be possible. If the test is positive this essentially 'infers' VITT (or HIT), and thus further "discriminatory" functional testing would be required. At first, 'standard' functional platelet activation assays normally used for HIT (i.e., with inclusion of heparin to identify augmented platelet activation and aggregation) is performed. If these "standard" assays are positive for $\mathrm{PF} 4 / \mathrm{H}$ antibody-mediated platelet activation and aggregation, the patient is identified as having HIT, exits the diagnostic pathway and is treated accordingly. If the patient is found to be negative for $\mathrm{PF} 4 / \mathrm{H}$ antibodymediated platelet activation and aggregation by standard assays, then a modified heparin induced platelet aggregation (HIPA) assay is performed, according to guidance from the Greinacher laboratory. A positive finding in the modified HIPA assay would identify VITT, and the patient managed according to a VITT management plan. An alternative negative finding in the modified HIPA assay would exclude VITT, and thus heparin treatment would instead be possible. Also notable within the guideline is a requirement to identify the presence of thrombocytopenia and/or thrombosis at the start of the process. It is important to consider this guidance within a historical context, since more recent evidence suggests that not all VITT cases will necessarily initially present with either thrombocytopenia or thrombosis (discussed later). Also, D-dimer testing did not feature in the algorithm, although this testing was recommended in the text of the manuscript. Nevertheless, the test for "PF4/H" antibodies was the key discriminator for the presence of VITT (or HIT), and a negative result provided an exit point for treatment. Again, this should be considered within a historical context, and it is now known that there is a small possibility of false negative PF4/H ELISA test results [7]. But perhaps most striking in the GTH guideline is the requirement for functional testing to assess platelet activation after a positive PF4/H ELISA assay result.

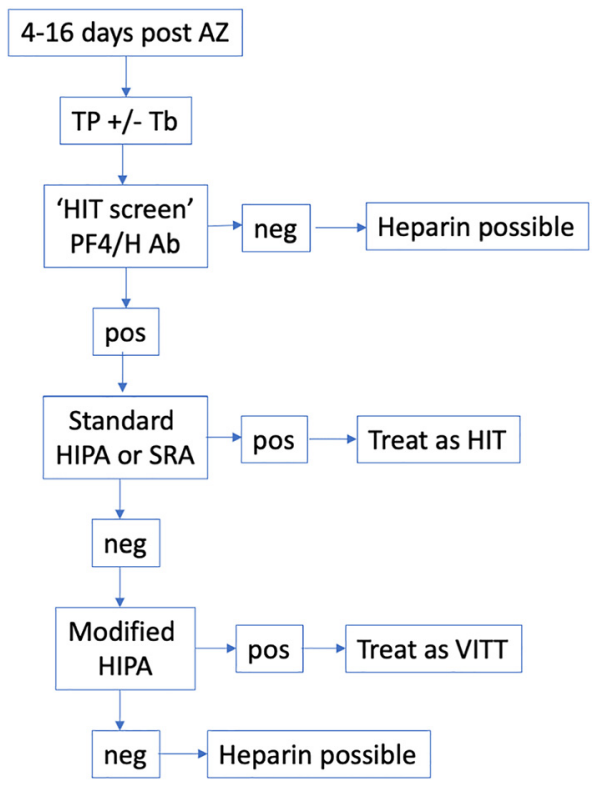

Figure 1: The VITT diagnostic pathway proposed by the Society for Thrombosis and Haemostasis Research (GTH) [22].

Ab, antibody; AZ, AstraZeneca (COVID-19 vaccine); H, heparin; HIPA, heparin induced platelet aggregation; HIT, heparin induced thrombocytopenia (with thrombosis); neg, negative; PF4, platelet factor 4; pos, positive; SRA, serotonin release assay; TP, thrombocytopenia; Tb, thrombosis; VITT, vaccine induced thrombotic thrombocytopenia. 
The UK group also published an algorithm in their NEJM paper [11], as partly summarized in Figure 2 for the diagnostic pathway. Again, this was based on their experience with $A Z$ vaccination. The post vaccination timeframe was longer than that of the GTH guideline, and also advocated early involvement of a hematologist. Again, presence of thrombosis and/or thrombocytopenia created the entry point for the pathway. A recommendation for routine coagulation tests, comprising prothrombin time (PT), activated partial thromboplastin time (APTT), fibrinogen and D-dimer provided the deflection point for likelihood of VITT (D-dimer >4,000 fibrinogen equivalent units (FEU), low or normal fibrinogen, and no alternate diagnosis) or not VITT (D-dimer <2,000 FEU, normal PT/APTT/ fibrinogen). This was sufficient to initiate differential treatment, at which time a "HIT ELISA" would be performed on suspected VITT cases. A negative result would trigger a review of diagnosis and treatment, or consideration of alternative investigations, including functional tests. A positive test result would justify continued treatment as VITT.

These initial UK guidelines no doubt provided the basis for further guidance by means of "living documentation"

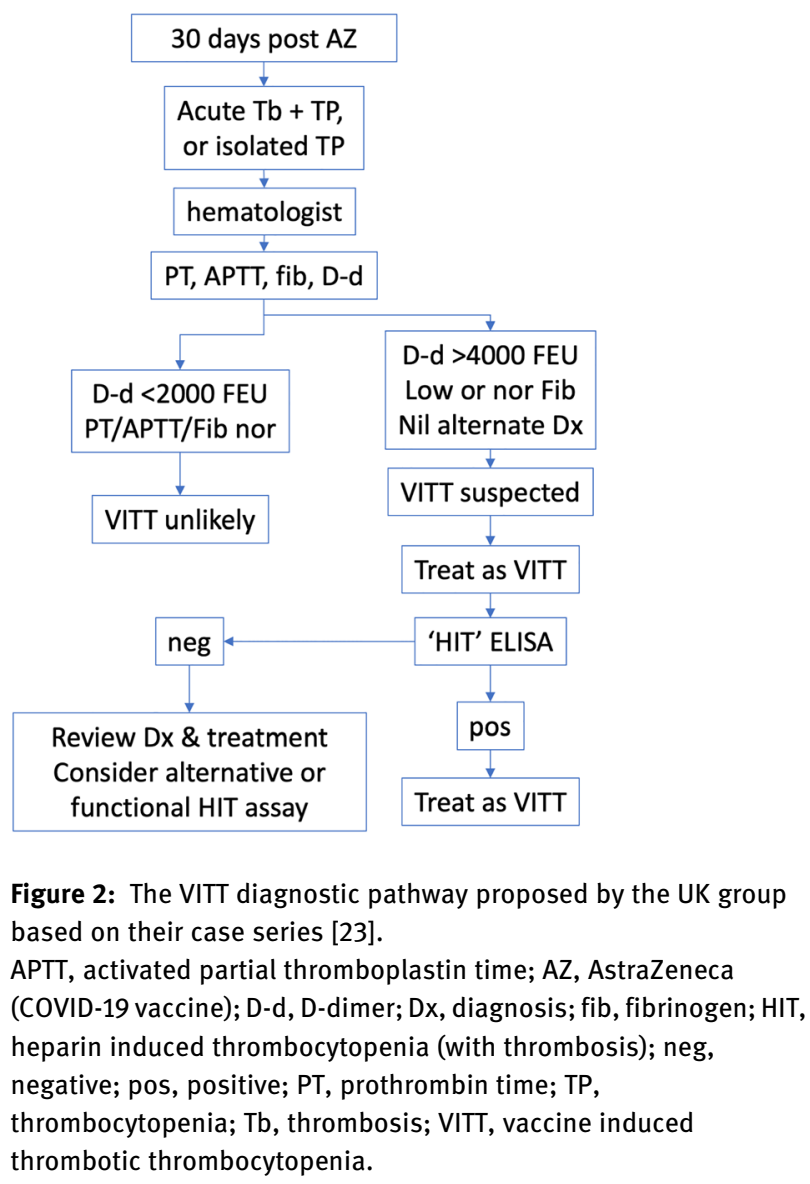

(meaning regularly updated) by the UK Expert Haematology Panel (EHP) focused on VITT [23]. Although the main aim of this expert guidance is on management of patients with VITT, there is a summary of VITT case definitions, which derives from another publication in NEJM [24], and as summarized in Table 1, as well as an investigative algorithm, which is similar to that shown in Figure 2. Also provided on the guidance is advice about ELISA testing, storing samples, sending samples for whole genome testing, presumably to help identify any potential genetic predeterminants for VITT development, and guidance about future vaccination.

Additional guidance is available from the National Institute for Health and Care Excellence (NICE), whose role is "to improve outcomes for people using the NHS and other public health and social care services" [25]. However, the guidelines, published on July 29th, and available online [26], are noted to be adapted from the UK EHP's guidance.

The Italian Society for the Study of Haemostasis and Thrombosis (SISET) has produced guidance for the management of cerebral and splanchnic vein thrombosis (CSVT or SVT) associated with thrombocytopenia in people previously vaccinated with $\mathrm{AZ}$ or other adenovirus-based vaccines [27]. This includes an algorithm that identifies possible VITT with symptoms within 30 days of vaccination, recommending to

Table 1: VITT Case definition criteria from the UK EHP.

\begin{tabular}{|c|c|}
\hline $\begin{array}{l}\text { VITT case defini- } \\
\text { tion criteria }\end{array}$ & $\begin{array}{l}\text { 1. Onset of symptoms } 5-30 \text { post COVID-19 vac- } \\
\text { cine (or up to } 42 \text { days if isolated DVT/PE) } \\
\text { 2. Presence of thrombosis } \\
\text { 3. Thrombocytopenia } \\
\text { (platelet count }<150 \times 10^{9} / \mathrm{L} \text { ) } \\
\text { 4. D dimer }>4,000 \mu \mathrm{g} / \mathrm{mL}(\mathrm{FEU} \text { ) } \\
\text { 5. Positive anti-PF } 4 \text { antibody ELISA assay }\end{array}$ \\
\hline Definite VITT & Meets all five criteria \\
\hline Probable VITT & $\begin{array}{l}\text { D dimer }>4,000 \mu \mathrm{g} / \mathrm{mL} \text { FEU but one criterion not } \\
\text { fulfilled (timing, thrombosis, thrombocytopenia, } \\
\text { anti-PF4 antibodies) or D dimer unknown or } \\
2,000-4,000 \mu \mathrm{g} / \mathrm{mL} \text { FEU with all other criteria } \\
\text { present }\end{array}$ \\
\hline Possible VITT & $\begin{array}{l}\text { D dimer unknown or } 2,000-4,000 \mu \mathrm{g} / \mathrm{mL} \mathrm{FEU} \\
\text { with one other criterion not fulfilled, or two other } \\
\text { criteria not fulfilled (timing, thrombosis, } \\
\text { thrombocytopenia, anti-PF4 antibodies) }\end{array}$ \\
\hline VITT Unlikely & $\begin{array}{l}\text { Platelet count }<150 \times 10^{9} / \mathrm{L} \text { without thrombosis } \\
\text { with } \mathrm{D} \text { dimer }<2,000 \mu \mathrm{g} / \mathrm{mL} \mathrm{FEU} \text {, or thrombosis } \\
\text { with platelet count }>150 \times 10^{9} / \mathrm{L} \text { and } \mathrm{D} \text { dimer } \\
<2,000 \mu \mathrm{g} / \mathrm{mL} \mathrm{FEU} \text {, regardless of anti-PF } 4 \text { anti- } \\
\text { body result, and/or alternative diagnosis more } \\
\text { likely }\end{array}$ \\
\hline
\end{tabular}

DVT, deep vein thrombosis; PE, pulmonary embolism; FEU, fibrinogen equivalent units; PF4, platelet factor 4; EHP, Expert Haematology Panel. 
perform testing for platelet count, international normalized ratio (INR), APTT, fibrinogen, D-dimer, an anti-PF4 assay, and HIPA if positive. Subsequent treatment is then dependent on the platelet count, as well as on results of the anti-PF4 assay.

The Spanish Federation of Medical and Scientific Associations (FACME) has also recently published some guidelines for diagnosis and treatment of VITT manifesting as cerebral venous sinus thrombosis (CVST) [28], with the diagnostic pathway summarized in Figure 3. Apart from identification of CVST within three weeks of vaccination with adenovirus-based vaccines, the presence of thrombocytopenia (or interestingly, a 50\% decrease in baseline platelet count) provides the entry point in the pathway, with suggestion for standard management but also continued monitoring of clinical signs and also blood counts for those without. For those with thrombocytopenia (or $50 \%$ decrease from a recently obtained previous value), a PF4 antibody test was recommended, and for positive cases to be managed as VITT. For PF4 antibody negative cases, D-dimer testing was recommended, and if value was $>4 \times$ the upper limit of normal (ULN), these cases should still be managed as VITT. If D-dimer was $<4 \times$ ULN, but no alternative explanation is available for concomitant thrombocytopenia, then these cases should also still be managed as VITT. Nonetheless, when alternative explanations are available for thrombocytopenia, then the case could be managed as per standard process, but with continued monitoring of clinical signs and blood counts.

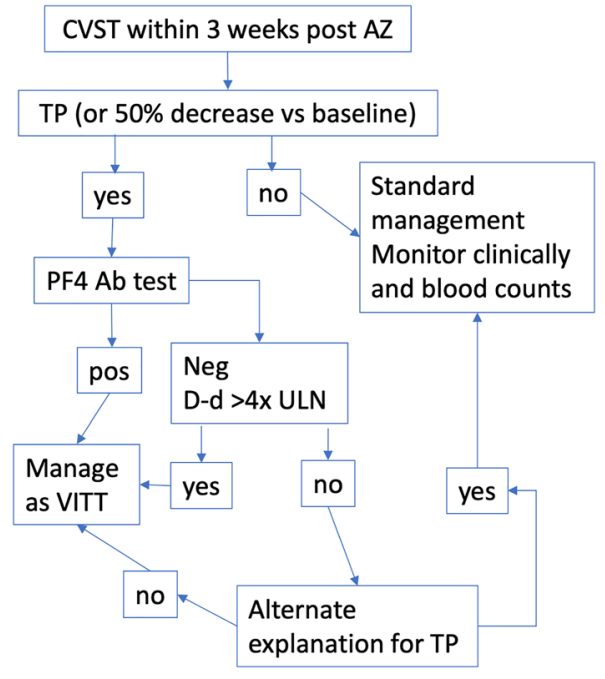

Figure 3: The VITT diagnostic pathway proposed by the Spanish Federation of Medical and Scientific Associations (FACME) [28]. $A b$, antibody; AZ, AstraZeneca (COVID-19 vaccine); neg, negative; PF4, platelet factor 4; pos, positive; TP, thrombocytopenia; ULN, upper limit of normal; VITT, vaccine induced thrombotic thrombocytopenia.

\section{Guidelines from North America}

As noted, VITT can also occur subsequent to the JJ vaccine $[7,12,13]$. One living document outlining both the identification and treatment of VITT is provided as an online resource from the American Society of Hematology [29], although they preferentially use the term TTS. Although an algorithm is not provided, like the UK EHP, the ASH guidance provides advice on "definitive diagnosis" of TTS, as well as its potential exclusion, as summarized in Table 2 . Given this is a living document, there is recognition in the most recent version of the guidance that a normal platelet count may not exclude VITT/TTS, since this may represent an early stage of the disease. A separate online resource is available from a Canadian group [30]. The guidance in relation to testing is summarized in Figure 4. The document has a May 7, 2021, publication date, and starts with

Table 2: TTS Case definition criteria from the American Society of Hematology.

Definitive diagnosis of TTS 1. COVID vaccine 4-42 days prior (must meet all five criteria) to symptom onset (a)

2. Any venous or arterial thrombosis (often cerebral or abdominal)

3. Thrombocytopenia (platelet count $\left.<150 \times 10^{9} / \mathrm{L}\right)(\mathrm{b})$

4. Positive PF4 “HIT" (heparin-induced thrombocytopenia) ELISA

5. Markedly elevated D-dimer ( $>4$ times upper limit of normal)

a) "To date, TTS appears far more likely following AstraZeneca/Johnson and Johnson adenoviral vaccines than Moderna/Pfizer mRNA vaccines"

b) "A patient who presents with thrombosis and a normal platelet count post-vaccination might be in an early stage of TTS. Continued assessment for development of thrombocytopenia/ $T$ S is required"

Initial laboratory work-up: $1 . \quad$ CBC with platelet count* and peripheral smear

2. PF4-ELISA (HIT assay); draw blood prior to any therapies

3. Fibrinogen and D-dimer

(*a normal platelet count is less concerning for TTS)

TTS is excluded if: $\quad$ PF4 ELISA is negative and there is no thrombocytopenia

Likely ITP if: $\quad$ Thrombocytopenia but no thrombosis and negative PF4 ELISA

CBC, complete blood count; HIT, heparin induced thrombocytopenia; ITP, immune thrombocytopenia purpura; PF4, platelet factor 4; TTS, thrombosis with thrombocytopenia syndrome. 
assessment of patient symptoms reflective of potential thrombosis or bleeding, between 4 and 28 days after vaccination, with complete blood count $(\mathrm{CBC})$ being the initial recommended blood test. Thrombocytopenia (i.e., platelet count $<150 \times 10^{9} / \mathrm{L}$ ) represents the deflection point between further testing or not; if present, D-dimer, a blood film, and diagnostic imaging based on clinical suspicion, is then recommended. The composite of these then decides the further progression, which includes "HIT ELISA testing" in cases "presumptive of VITT".

Another source of information for those wishing to learn about VITT/TTS is an online resource written by key North American researchers Warkentin and Cuker [31]. Again, this document is regularly reviewed and updated.

\section{Guidelines from Australia (and New Zealand)}

Diagnostic and management guidelines for VITT from Australia and New Zealand have been prepared by a group of experts on behalf of the Thrombosis and Haemostasis Society of Australia and New Zealand (THANZ). The main document is another "living" document, regularly updated, initially with weekly meetings of the THANZ vaccine thrombocytopenia advisory working group, comprising some 25 members, including two of the authors of this review. Available online [32], the resource was first provided

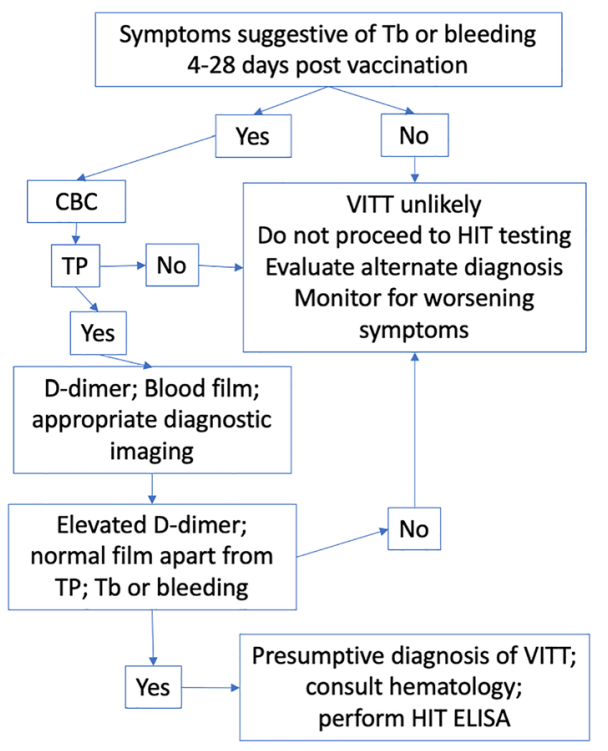

Figure 4: The VITT diagnostic pathway proposed by the Ontario COVID-19 Science Advisory Table [30].

CBC, complete blood count; Tb, thrombosis; TP, thrombocytopenia; VITT, vaccine induced thrombotic thrombocytopenia. in April, 2021, and last updated in mid-August. Some key elements of the guidance, and some sample Australian cases of VITT have been recently published [21, 33]. It can be noted that $\mathrm{AZ}$ has been the only adenovirus-based vaccine used in Australia so far. No adenovirus-based vaccine is used in New Zealand; however, THANZ membership inclusive of New Zealand participation was identified as important since there is potential for cross travel of $A Z$ vaccinated individuals from Australia and abroad, who may have also received adenovirus-based vaccines. A summary of the diagnostic pathway for VITT is shown in Figure 5. Recognizing this is a recently updated version, some key points can be noted, as informed based on local Australian cases: (a) extension of vaccine exposure out to 42 days; (b) recognition that thrombocytopenia may not be evident in VITT, either because of early stage disease or high baseline pre-vaccination platelet counts, not always available in VITT investigations; (c) inclusion of high D-dimers ( $>5 \times$ ULN) as a marker of VITT; (d) a different pathway of testing for PF4 antibodies/functional testing based on presence/absence of thrombosis - thus, (i) no thrombosis would lead to PF4 ELISA testing, but functional testing would only progress in ELISA positive cases, (ii) presence of thrombosis would lead to concurrent anti-PF4 ELISA and functional testing. This differential was mostly aimed at managing the resource intensive functional testing to those most warranting testing based on management considerations. Additional guidance on management according to findings is also provided [21, 32]. From a laboratory testing approach, the THANZ strategy represents perhaps the most comprehensive and nationally coordinated approach, reflective of strong local research presence and includes functional testing by several tests, namely SRA, by a novel flow cytometry-based method, and by whole blood Multiplate testing. In addition to guidance prepared for Hematologists, several THANZ members have also helped prepare a number of other guidance documents, including a general VITT Multidisciplinary Guideline for Doctors [34]. Similar to the UK EHP, the THANZ also has criteria for diagnosis/exclusion of VITT, with the current scheme summarized in Table 3. Again, this classification is reflective of the Australian VITT cohort, currently in excess of 100 cases. Comprehensive data on these VITT cases from Australia is currently being prepared for publication.

\section{Guidelines from the ISTH}

As the premier body representing international work in the field of thrombosis and hemostasis, the International 


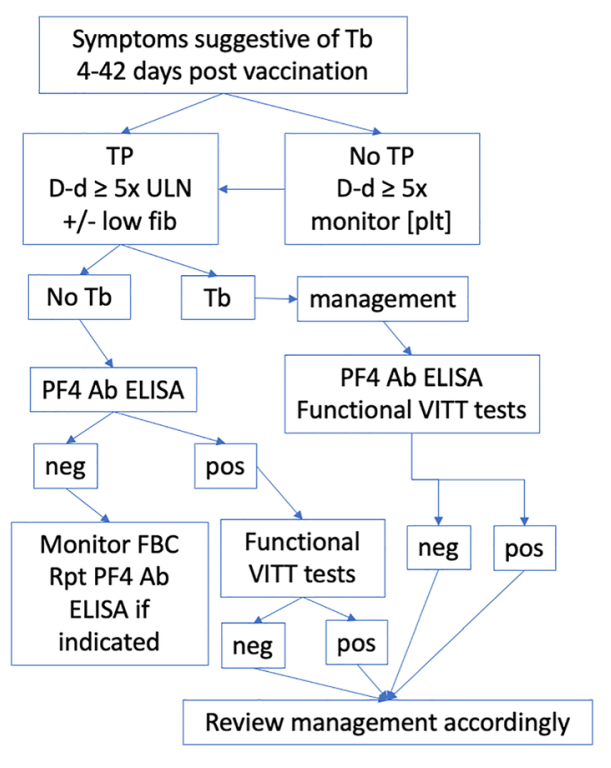

Figure 5: The VITT diagnostic pathway proposed by THANZ based on the Australian case series [21].

$A b$, antibody; D-d, D-dimer; ELISA, enzyme linked immunosorbent assay; FBC, full blood count; fib, fibrinogen; neg, negative; PF4, platelet factor 4; pos, positive; TP, thrombocytopenia; Tb, thrombosis; VITT, vaccine induced thrombotic thrombocytopenia.

Society on Thrombosis and Haemostasis (ISTH) has also prepared guidance on VITT identification and management, inclusive of a flow chart [35]. The diagnostic pathway is summarized in Figure 6. It also needs to be recognized that the flow chart was produced quite early in the VITT story, and so is due for revision. At current understanding of VITT, 28 days post-vaccination may be too restrictive to permit exclusion of VITT. Also, thrombosis is not always apparent on first presentation, and may arise subsequently. Third, thrombocytopenia is not always present on first presentation, and may or may not arise subsequently. Finally, there is a small incidence of false ELISA negative in some cases of VITT, which would require either testing with another ELISA assay, or by functional assay(s), to truly exclude VITT.

\section{Discussion}

In this review, we have identified and detailed several diagnostic guidelines for VITT/TTS. It can be noted that all the guidelines differ in some aspects of the suggested approach. This may then determine different rates of VITT detection or exclusion, and indeed the potential identification of different VITT cohorts. In the early VITT publications, most patients presented with unusual site thromboses, including CVST or SVT, but this may in part
Table 3: VITT Case classification criteria from THANZ.

\begin{tabular}{|c|c|}
\hline \multicolumn{2}{|c|}{ Classification prior to specific VITT testing } \\
\hline Suspected & $\begin{array}{l}\text { VITT is suspected if ( } 1) \text { the platelet count is } \\
<150 \times 10^{9} / L \text { AND either }(2) D \text {-dimers are elevated } \\
(5 \times \text { ULN) OR (3) fibrinogen is reduced }\end{array}$ \\
\hline Probable & $\begin{array}{l}\text { VITT is probable if there is evidence of thrombosis } \\
\text { in suspected VITT }\end{array}$ \\
\hline Possible & $\begin{array}{l}\text { VITT is possible if there is no evidence of throm- } \\
\text { bosis in suspected VITT }\end{array}$ \\
\hline Less likely & $\begin{array}{l}\text { VITT is less likely if the platelet count is }>150 \times 10^{9} / \\
\text { L, but } D \text {-dimers are elevated or fibrinogen is } \\
\text { reduced }\end{array}$ \\
\hline Much less likely & $\begin{array}{l}\text { VITT is much less likely if repeated platelet counts } \\
\text { are }>150 \times 10^{9} / \mathrm{L} \text {, D-dimers are not elevated AND } \\
\text { fibrinogen is normal }\end{array}$ \\
\hline \multicolumn{2}{|c|}{ Classification following specific VITT testing } \\
\hline Confirmed & $\begin{array}{l}\text { VITT is confirmed by positive "VITT" ELISA, AND } \\
\text { positive "VITT" functional testing in cases of sus- } \\
\text { pected VITT }\end{array}$ \\
\hline Strongly & VITT is strongly supported by strong positive "VITT" \\
\hline supported & $\begin{array}{l}\text { ELISA testing, or by "VITT" functional testing, in } \\
\text { cases of suspected VITT }\end{array}$ \\
\hline Possible & $\begin{array}{l}\text { VITT remains possible in suspected VITT whose } \\
\text { "VITT" ELISA is positive, but functional "VITT" } \\
\text { testing is negative }\end{array}$ \\
\hline Unsupported & $\begin{array}{l}\text { VITT is unsupported by negative "VITT" ELISA, and } \\
\text { negative functional "VITT" testing, in cases of } \\
\text { suspected VITT }\end{array}$ \\
\hline Unclassified & $\begin{array}{l}\text { 1. Serious thrombosis (likely VITT) with negative } \\
\text { ELISA and functional testing } \\
\text { 2. Normal platelet count, without raised D-dimer, } \\
\text { but thrombosis within time frame: ELISA and } \\
\text { functional positive }\end{array}$ \\
\hline
\end{tabular}

ELIZA, enzyme linked immunosorbent assay; ULN, upper limit of normal; "VITT" ELISA represents a platelet factor 4 (PF4) ELISA; "VITT" functional testing infers one or more of: "VITT" modified serotonin release assay (SRA), flow cytometry platelet activation assay, Multiplate whole blood aggregation assay.

reflect ascertainment bias. Thus, thrombosis in VITT can also occur in standard sites, including deep vein thrombosis (DVT) and pulmonary embolism (PE) [24, 36]. That lack of initial detection of these cases could simply have reflected case ascertainment bias is now recognized [37], given DVT and PE are otherwise common thrombosis presentations outside of the VITT setting and may not have been considered as a protentional consequence of vaccination. The initial and subsequent laboratory testing recommendations also differ between guidelines. To some extent, such differences can also reflect the timing of release of the guidelines, with later documents reflecting improved knowledge about VITT. Let us take, for example, the presence of thrombocytopenia. In early guidelines, absence of thrombocytopenia was taken to mean an absence of VITT. This was based on the clear reported 


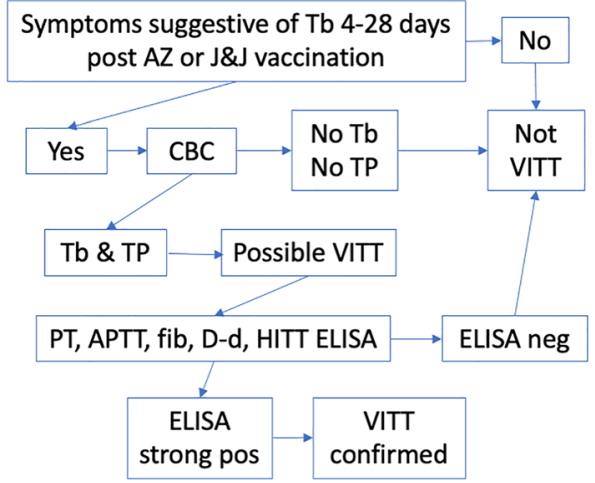

Figure 6: The VITT diagnostic pathway proposed by ISTH [35]. AZ, AstraZenica vaccine; D-d, D-dimer; ELISA, enzyme linked immunosorbent assay; CBC, complete blood count; fib, fibrinogen; neg, negative; HITT, heparin induced thrombocytopenia with thrombosis; J\&J, Johnson \& Johnson vaccine; pos, positive; TP, thrombocytopenia; Tb, thrombosis; VITT, vaccine induced thrombotic thrombocytopenia.

findings of thrombocytopenia in all cases of reported VITT to date at that time [7]. To some extent, this may also reflect case ascertainment bias. We know from studies on HIT, that not all cases of HIT present with thrombocytopenia, such as for example in patients with a baseline high platelet count (i.e., over 300-400 × 109/L). Indeed, built into the 4T HIT probability score, instead of thrombocytopenia, a 50\% drop in platelet count can also be used to infer on the likelihood of HIT. For a patient with high baseline count (e.g., $>350 \times 10^{9} / \mathrm{L}$ ), a drop of $50 \%$ can still reflect a platelet count above $150 \times 10^{9} / \mathrm{L}$. However, in VITT investigations, being community-based cohorts of otherwise generally healthy individuals, a pre-vaccination platelet count is rarely available. Thus, a lack of thrombocytopenia should not be used to exclude VITT, and patients with other signs pointing to VITT should have their platelet counts monitored for subsequent reduction.

The other main initial marker of suspected VITT is the D-dimer level, which have generally been $>5 \times$ ULN. To our minds, a highly raised D-dimer may reflect a more consistent finding in VITT that thrombocytopenia. Only some of the guidelines place sufficient emphasis on the importance of D-dimer in the initial triaging of cases for investigation. Indeed, a patient presenting with thrombocytopenia but normal or only mildly elevated D-dimer (i.e., $<2 \times \mathrm{ULN}$ ) is more likely to have immune thrombocytopenic purpura (ITP), than VITT.

Although fibrinogen testing is useful, it has a less important role in triaging patient investigation than either platelet count or D-dimer. Historically, some $70 \%$ of VITT patients seem to have reduced levels of fibrinogen, probably due to its consumption within the thrombotic process
[7]. Nonetheless, fibrinogen monitoring is important also for assessing bleeding risk, particularly if also associated with severe thrombocytopenia. Monitoring of fibrinogen, along with D-dimer and platelet count, may also help identify improvements due to treatment (e.g., IVIG and anticoagulation) [38].

The testing of anti-PF4 antibodies by ELISA is crucial to a correct diagnosis or exclusion of VITT, but again is not fool-proof. It is interesting that some of the guidelines do not specify that anti-PF4 antibodies should be evaluated by ELISA, and not by say other rapid anti-PF4 antibodies normally used to investigate HIT, and which tend to be negative in VITT [7]. Also, some guidelines actually mention "HIT ELISA" instead of anti-PF4-ELISA, which may confuse some non-experts. It has also been shown that anti-PF4 antibody ELISA assays modified for VITT testing will show increased sensitivity and specificity for VITT [7].

It is also interesting that not all guidelines provide strong support for functional VITT testing. Strong support for specific functional VITT testing, including VITT modified assays, is provided by the Greinacher group [9], as well as the associated GTH guidelines [22], and the THANZ guidelines [21, 32]. The lack of consensus to date in this aspect is at least partially attributable to the fact that positivity in functional tests like SRA seems to be influenced by certain important technical aspects, such as the addition of FP4 to the test reagents with the purpose of enhancing the diagnostic sensitivity [7]. Also, functional assays are not as widely available as anti-PF4 ELISA assays, and are more complex to perform. In summation, we would note here that the situation is complex. One the one hand, a background rate of anti-PF4 antibodies (albeit generally weak) of up to 5\% can be observed in otherwise healthy individuals according to the studies of Thiele et al. [39] \& Sørvoll et al. [40]. Co-incidental (mild) thrombocytopenia and thrombosis may also arise in otherwise healthy individuals unrelated to vaccine use. Thrombocytopenia with normal or only mildly elevated D-dimer may instead be pointing to ITP. Naturally, the composite of all three events (thrombocytopenia, thrombosis, highly increased D-dimer values) post-adenovirus based COVID-19 vaccination, in the right timeframe, together with positive anti-PF4 ELISA, does strongly suggest VITT, and may be sufficient for clinical diagnosis, especially if functional testing is not available. On the other hand, functional testing provides yet additional evidence, and is perhaps the most definitive test for VITT from a pathophysiological perspective. Two of the authors are members of the THANZ Advisory Group mentioned in this paper, and we are of course influenced by our own Australian experience, which has actually evaluated a number of different functional assays [21]. 
In terms of post vaccination timeframe for VITT, the early reports of VITT within 5-16 days [9], 7-10 days [10], 6-24 days [11], biased to presentations within the early cases series, have continued to expand, given presentations outside those timeframes. The latest iteration of the THANZ guidelines indicate a timeframe of 4-42 days $[27,29]$ based on presentations of Australian VITT cases, and the latest EHP guidance indicates that "cases usually present 5-30 days after vaccination" but "that DVT and PE can present up to 42 days after vaccination" and that DVT probably "develops subclinically between days 5-30" [19].

\section{Conclusions}

As the specific knowledge on the pathogenesis, clinical and laboratory presentation of VITT grows, the availability, applicability and completeness of diagnostic guidelines will expectedly evolve in parallel, though the accuracy will remain limited, due to the variegated, often subtle presentations of VITT. Notably, the frequency of both vaccineassociated thrombocytopenia and vaccine-associated ITP has been assessed in a large Scottish population-based study, which included people who received both AstraZeneca and Pfizer vaccines. This study revealed that the risk of developing ITP was reported at around 1.13 per 100,000 recipients of $\mathrm{AZ}$ adenovirus-based vaccine, especially between 1 and 3 weeks post vaccination, and without any further evidence of thrombosis [41]. Similarly, although it is now already ascertained that anti-PF4 antibodies develop in a significant proportion of adenovirus-based COVID-19 vaccine recipients [39, 40, 42], they are very rarely associated with thrombotic episodes and, importantly, their levels also seem to correlate with those of anti-SARS-CoV-2 neutralizing antibodies [42], perhaps suggesting that baseline anti-SARS-CoV-2 positive individuals should preferably be administered with non-adenovirus-based formulations of COVID-19 vaccines. Therefore, an accurate diagnosis can only be reached when multiple abnormalities are integrated, to define a highly suggestive clinical and laboratory picture. What can hence be summarized so far from the existing documents is that diagnostic algorithms should encompass (at least) a complete blood cell count for obtaining the platelet number, along with assessment of plasma fibrinogen and D-dimer, eventually followed by anti-PF4-based ELISA, and perhaps reserving functional platelet assays to diagnostically challenging cases [21, 32, 43], or for research investigation.
Acknowledgments: The authors acknowledge the contribution of the THANZ VITT Advisory Group/Working Party in the development of the THANZ guidance.

Research funding: None declared.

Author contributions: EJF was responsible for planning the review, and wrote the original draft of this manuscript. All authors contributed content to the review, helped revise the manuscript and approved its submission. The authors acknowledge the contribution of the THANZ VITT Advisory Group/Working Party in the development of the THANZ guidance [21, 32]. The views expressed herein are those of the authors and are not necessarily those of NSW Health Pathology, or the University of Verona. All authors have accepted responsibility for the entire content of this manuscript and approved its submission.

Competing interests: Authors state no conflict of interest. Informed consent: Not applicable.

Ethical approval: Not applicable.

Data availability: Data sharing not applicable to this review article.

\section{References}

1. COVID-19 Dashboard by the Center for Systems Science and Engineering (CSSE) at Johns Hopkins University (JHU). Available from: https://www.arcgis.com/apps/dashboards/ bda7594740fd40299423467b48e9ecf6 [Accessed 16 Oct 2021].

2. COVID-19 vaccines. Available from: https://en.wikipedia.org/ wiki/COVID-19_vaccine [Accessed 16 Sep 2021].

3. World Health Organisation (WHO). COVID-19 vaccine tracker and landscape. Available from: https://www.who.int/publications/ $\mathrm{m} /$ item/draft-landscape-of-covid-19-candidate-vaccines [Accessed 24 Sep 2021].

4. CDC recommends use of Johnson \& Johnson's Janssen COVID-19 vaccine resume. Available from: https://www.cdc.gov/ coronavirus/2019-ncov/vaccines/safety/JJUpdate.html [Accessed 16 Sep 2021].

5. EMA raises awareness of clinical care recommendations to manage suspected thrombosis with thrombocytopenia syndrome. Available from: https://www.ema.europa.eu/en/news/ema-raisesawareness-clinical-care-recommendations-manage-suspectedthrombosis-thrombocytopenia [Accessed 16 Sep 2021].

6. COVID-19 vaccine weekly safety report - 12-08-2021. Available from: https://www.tga.gov.au/periodic/covid-19-vaccineweekly-safety-report-12-08-2021 [Accessed 16 Sep 2021].

7. Favaloro EJ. Laboratory testing for suspected COVID-19 vaccineinduced (immune) thrombotic thrombocytopenia. Int J Lab Hematol 2021;43:559-70.

8. Greinacher A, Thiele T, Warkentin TE, Weisser K, Kyrle P, Eichinger S. A prothrombotic thrombocytopenic disorder resembling heparin-induced thrombocytopenia following coronavirus-19 vaccination. Res Sq 2021. https://doi.org/10. 21203/rs.3.rs-362354/v1. 
9. Greinacher A, Thiele T, Warkentin TE, Weisser K, Kyrle PA, Eichinger S. Thrombotic thrombocytopenia after ChAdOx1 nCov19 vaccination. N Engl J Med 2021;384:2092-101.

10. Schultz NH, Sørvoll IH, Michelsen AE, Munthe LA, Lund-Johansen F, Ahlen MT, et al. Thrombosis and thrombocytopenia after ChAdOx1 nCoV-19 vaccination. N Engl J Med 2021;384:2124-30.

11. Scully M, Singh D, Lown R, Poles A, Solomon T, Levi M, et al. Pathologic antibodies to platelet factor 4 after ChAdOx1 nCoV-19 vaccination. N Engl J Med 2021;384:2202-11.

12. Muir KL, Kallam A, Koepsell SA, Gundabolu K. Thrombotic thrombocytopenia after Ad26.COV2.S vaccination. $\mathrm{N}$ Engl J Med 2021;384:1964-5.

13. See I, Su JR, Lale A, Woo EJ, Guh AY, Shimabukuro TT, et al. US case reports of cerebral venous sinus thrombosis with thrombocytopenia after Ad26.COV2.S Vaccination, March 2 to April 21, 2021. J Am Med Assoc 2021;325:2448-56. e217517.

14. Sangli S, Virani A, Cheronis N, Vannatter B, Minich C, Noronha S, et al. Thrombosis with thrombocytopenia after the messenger RNA-1273 vaccine. Ann Intern Med 2021;174:1480-82.

15. Pishko AM, Cuker A. Thrombosis after vaccination with messenger RNA-1273: is this vaccine-induced thrombosis and thrombocytopenia or thrombosis with thrombocytopenia syndrome? Ann Intern Med 2021;174:1468-9.

16. Favaloro EJ, McCaughan G, Pasalic L. Clinical and laboratory diagnosis of heparin induced thrombocytopenia: an update. Pathology 2017;49:346-55.

17. Favaloro EJ, Mohammed S, Donikian D, Kondo M, Duncan E, Yacoub O, et al. A multicentre assessment of contemporary laboratory assays for heparin induced thrombocytopenia. Pathology 2021;53:247-56.

18. Hvas AM, Favaloro EJ, Hellfritzsch M. Heparin-induced thrombocytopenia: pathophysiology, diagnosis and treatment. Expet Rev Hematol 2021;14:335-46.

19. Huynh A, Kelton JG, Arnold DM, Daka M, Nazy I. Antibody epitopes in vaccine-induced immune thrombotic thrombocytopaenia. Nature 2021;596:565-9.

20. Vayne C, Rollin J, Gruel Y, Pouplard C, Galinat H, Huet O, et al. PF4 immunoassays in vaccine-induced thrombotic thrombocytopenia. N Engl J Med 2021;385:376-8.

21. Chen VM, Curnow JL, Tran HA, Choi PY. Australian and New Zealand approach to diagnosis and management of vaccineinduced immune thrombosis and thrombocytopenia. Med J Aust 2021;215:245-9.

22. Oldenburg J, Klamroth R, Langer F, Albisetti M, von Auer C, Ay C, et al. Diagnosis and management of vaccine-related thrombosis following AstraZeneca COVID-19 vaccination: guidance statement from the GTH. Hämostaseologie 2021;41:184-9.

23. Guidance produced by the Expert Haematology Panel (EHP) focussed on Vaccine induced Thrombosis and Thrombocytopenia (VITT). Available from: https://b-s-h.org.uk/about-us/news/ guidance-produced-by-the-expert-haematology-panel-ehpfocussed-on-vaccine-induced-thrombosis-andthrombocytopenia-vitt/ [Accessed 16 Sep 2021].

24. Pavord S, Scully M, Hunt BJ, Lester W, Bagot C, Craven B, et al. Clinical features of vaccine-induced immune thrombocytopenia and thrombosis. N Engl J Med 2021. https://doi.org/10.1056/ NEJMoa2109908.

25. National Institute for Health and Care Excellence (NICE). What we do. Available from: https://www.nice.org.uk/about/what-we-do [Accessed 16 Sep 2021].
26. National Institute for Health and Care Excellence (NICE). COVID-19 rapid guideline: vaccine-induced immune thrombocytopenia and thrombosis (VITT). Available from: https://www.nice.org.uk/ guidance/ng200 [Accessed 16 Sep 2021].

27. Gresele P, Marietta M, Ageno W, Marcucci R, Contino L, Donadini MP, et al. Management of cerebral and splanchnic vein thrombosis associated with thrombocytopenia in subjects previously vaccinated with Vaxzevria (AstraZeneca): a position statement from the Italian Society for the Study of Haemostasis and Thrombosis (SISET). Blood Transfus 2021;19:281-3.

28. FACME multidisciplinary working group on the management of cerebral venous sinus thrombosis associated with COVID-19 vaccination. Diagnostic and treatment recommendations from the FACME ad-hoc expert working group on the management of cerebral venous sinus thrombosis associated with COVID-19 vaccination. Neurologia 2021;36:451-61.

29. Thrombosis with thrombocytopenia syndrome (also termed vaccine-induced thrombotic thrombocytopenia). Available from: https://www.hematology.org/covid-19/vaccine-inducedimmune-thrombotic-thrombocytopenia [Accessed 17 Sep 2021].

30. Pai M, Chan B, Stall NM, Grill A, Ivers N, Maltsev A, et al. Vaccineinduced immune thrombotic thrombocytopenia (VITT) following adenovirus vector COVID-19 vaccination. Sci Brief Ontario Covid19 Sci Advis Table 2021;2. https://doi.org/10.47326/ocsat.2021. 02.17.2.0.

31. Warkentin TE, Cuker A. COVID-19: vaccine-induced immune thrombotic thrombocytopenia (VITT). Available from: https://www. uptodate.com/contents/covid-19-vaccine-induced-immunethrombotic-thrombocytopenia-vitt [Accessed 17 Sep 2021].

32. THANZ vaccine thrombocytopenia working group. Suspected VITT THANZ Advisory Statement for Haematologists. Available from: https://www.thanz.org.au/documents/item/591 [Accessed 17 Sep 2021].

33. Hocking J, Chunilal SD, Chen VM, Brighton T, Nguyen J, Tan J, et al. The first known case of vaccine-induced thrombotic thrombocytopenia in Australia. Med J Aust 2021;215:19-20.e1.

34. VITT multidisciplinary guideline for doctors. Available from: https://www.thanz.org.au/news/vitt-multidisciplinaryguideline-for-doctors [Accessed $17 \mathrm{Sep}, 2021$ ].

35. The ISTH releases interim guidance on vaccine-induced immune thrombotic thrombocytopenia (VITT). Available from: https:// www.isth.org/news/561406/The-ISTH-Releases-InterimGuidance-on-Vaccine-Induced-Immune-ThromboticThrombocytopenia-VITT-.htm [Accessed 17 Sep 2021].

36. Gabarin N, Patterson S, Pai M, Afzaal T, Nazy I, Sheppard Jl, et al. Venous thromboembolism and mild thrombocytopenia after ChAdOx1 nCoV-19 vaccination. Thromb Haemostasis 2021. https://doi.org/10.1055/a-1585-6182.

37. Favaloro EJ, Pasalic L. COVID-19 vaccine induced (immune) thrombotic thrombocytopenia (VITT)/thrombosis with thrombocytopenia syndrome (TTS): an update. Aust J Med Sci 2021;42:86-93.

38. Bourguignon A, Arnold DM, Warkentin TE, Smith JW, Pannu T, Shrum JM, et al. Adjunct immune globulin for vaccine-induced immune thrombotic thrombocytopenia. N Engl J Med 2021;385: 720-8.

39. Thiele T, Ulm L, Holtfreter S, Schönborn L, Kuhn SO, Scheer C, et al. Frequency of positive anti-PF4/polyanion antibody tests after COVID-19 vaccination with ChAdOx1 nCoV-19 and BNT162b2. Blood 2021;138:299-303. 
40. Sørvoll IH, Horvei KD, Ernstsen SL, Laegreid IJ, Lund S, Grønli RH, et al. An observational study to identify the prevalence of thrombocytopenia and anti-PF4/polyanion antibodies in Norwegian health care workers after COVID-19 vaccination. J Thromb Haemostasis 2021;19:1813-8.

41. Simpson CR, Shi T, Vasileiou E, Katikireddi SV, Kerr S, Moore E, et al. First-dose ChAdOx1 and BNT162b2 COVID-19 vaccines and thrombocytopenic, thromboembolic and hemorrhagic events in Scotland. Nat Med 2021;27:1290-7.
42. Terpos E, Politou M, Ntanasis-Stathopoulos I, Karalis V, Merkouri E, Fotiou D, et al. High prevalence of anti-PF4 antibodies following ChAd0x1 nCov-19 (AZD1222) vaccination even in the absence of thrombotic events. Vaccines 2021;9:712.

43. Rizk JG, Gupta A, Sardar P, Henry BM, Lewin JC, Lippi G, et al. Clinical characteristics and pharmacological management of COVID-19 vaccine-induced immune thrombotic thrombocytopenia with cerebral venous sinus thrombosis: a review. JAMA Cardiol 2021 Aug 10. https://doi.org/10.1001/jamacardio.2021.3444 [Epub ahead of print]. 